\title{
Characterization of Streptococcus pneumoniae isolates from Austrian companion animals and horses
}

\author{
Maximilian Ginders ${ }^{1}$, Michael Leschnik², Frank Künzel ${ }^{2}$, Doris Kampner ${ }^{2}$, Claudia Mikula ${ }^{3}$, Georg Steindl ${ }^{4}$, \\ Inga Eichhorn ${ }^{5}$, Andrea T. Feßler ${ }^{5}$, Stefan Schwarz ${ }^{5}$, Joachim Spergser ${ }^{1}$ and Igor Loncaric ${ }^{1 *}$ (D)
}

\begin{abstract}
Background: The aim of the present study was to investigate the genetic relatedness and the antimicrobial resistance profiles of a collection of Austrian Streptococcus pneumoniae isolates from companion animals and horses. A total of 12 non-repetitive isolates presumptively identified as S. pneumoniae were obtained during routinely diagnostic activities between March 2009 and January 2017.

Results: Isolates were confirmed as S. pneumoniae by bile solubility and optochin susceptibility testing, matrixassisted laser desorption-ionization-time of flight (MALDI-TOF) mass spectrometry and sequence analysis of a part recA and the $16 \mathrm{~S}$ rRNA genes. Isolates were further characterized by pneumolysin polymerase chain reaction (PCR) and genotyped by multilocus sequence typing (MLST). Antimicrobial susceptibility testing was performed and resistance genes were detected by specific PCR assays. All isolates were serotyped. Four sequence types (ST) (ST36, ST3546, ST6934 and ST6937) and four serotypes (3, 19A, 19F and 23F) were detected. Two isolates from twelve displayed a multidrug-resistance pheno- and genotype.

Conclusions: This study represents the first comprehensive investigation on characteristics of S. pneumoniae isolates recovered from Austrian companion animals and horses. The obtained results indicate that common human sero(23F) and sequence type (ST36) implicated in causing invasive pneumococcal disease (IPD) may circulate in dogs. Isolates obtained from other examined animals seem to be host-adapted.
\end{abstract}

Keywords: Companion animals, Horses, Multi-drug resistant, Streptococcus pneumoniae, Zoonosis

\section{Background}

Streptococcus pneumoniae is a major human pathogen that colonizes the upper respiratory tract and causes both life-threatening diseases such as pneumonia, sepsis and meningitis but also sinusitis and otitis in both children and adults [1]. S. pneumoniae is responsible for community-acquired respiratory tract infections in infants. S. pneumoniae infections of pregnant women may be associated with stillbirth and fetal death [2]. Several animal models have been used to study

\footnotetext{
*Correspondence: igor.loncaric@vetmeduni.ac.at

1 Department of Pathobiology, Institute of Microbiology, University

of Veterinary Medicine Vienna, Vienna, Austria

Full list of author information is available at the end of the article
}

Pneumococcus-associated pneumonia, meningoencephalitis and otitis [3, 4]. S. pneumoniae is assumed to be a human pathogen only. Nevertheless, there are established mouse and rat models for various S. pneumoniaecaused diseases [4]. Zooanthropogenic transmission of other streptococcal species is well documented, in particular for S. equi subsp. zooepidemicus, S. canis, S. suis, S. porcinus and S. phocae [5-9]. Pneumococci can easily exchange DNA in their natural habitat, the human mouth and throat. This environment is populated by several streptococcal species, which form a 'gene pool' out of which the pneumococci can recruit resistance genes. Gene transfer and mosaic genes have been intensively reported for S. pneumoniae and other streptococci [10]. While comprehensive data on human S. pneumoniae 
infections exists, there is still a lack of information on infections, carriage and zoonotic potential of this particular pathogen in pet and companion animals. Therefore, the aim of the present study was to investigate the genetic relatedness and the antimicrobial resistance pattern of a collection of Austrian S. pneumoniae isolates from infections of pet and companion animals as well as horses.

\section{Methods}

A total of 12 non-repetitive isolates, presumptively identified as S. pneumoniae, were obtained between 2009 and 2017 during diagnostic examinations at the Institute of Microbiology of the University of Veterinary Medicine, Vienna, Austria. The isolates were identified as S. pneumoniae using classical bacteriological methods and originated from guinea pigs $(\mathrm{n}=6)$, horses $(\mathrm{n}=3)$, a $\operatorname{dog}(\mathrm{n}=1)$ and pet rats $(\mathrm{n}=2)$. The annual isolation frequency of $S$. pneumoniae was inconstant. All isolates were stored in glycerol stocks at $-80{ }^{\circ} \mathrm{C}$. For the present study, isolates were re-grown on Mueller-Hinton Agar with 5\% sheep blood and on Improved II agar [Becton-Dickinson (BD), Heidelberg, Germany]. They were confirmed as S. pneumonia by bile solubility [19] and optochin susceptibility testing [11], matrix-assisted laser desorption-ionization-time of flight mass spectrometry (MALDI-TOF MS) (Bruker Daltonik), as well as sequence analyses of a part of the recA [12] and $16 \mathrm{~S}$ rRNA genes [13]. Isolates were further characterized by pneumolysin polymerase chain reaction (PCR) [14] and genotyped by multilocus sequence typing (MLST). MLST was carried out by PCR amplification and sequencing of seven housekeeping genes (aroE, gdh, gki, recP, spi, xpt, $d d l)$ as described previously [15]. Allelic profiles and sequence types (ST) were assigned using the MLST database hosted at http://pubmlst.org/spneumoniae/. Antimicrobial susceptibility testing was performed by agar disc diffusion according to the Clinical and Laboratory Standards Institute (CLSI) [16, 17] including oxacillin, tetracycline, doxycycline, erythromycin, clindamycin, chloramphenicol, vancomycin, trimethoprim-sulfamethoxazole, enrofloxacin, marbofloxacin and linezolid (all from Becton-Dickinson, Heidelberg, Germany). With respect to the resistance phenotype, resistance genes were detected by previously described PCRs applying specific primers for tet(M), $\operatorname{erm}(\mathrm{B}), \operatorname{mef}(\mathrm{A}), \operatorname{mef}(\mathrm{E})$, $m s r(\mathrm{E}) c_{\mathrm{pC} 194}, c_{\mathrm{p}} t_{\mathrm{pC} 221}$ and $c a t_{\mathrm{pC} 223}[18-21]$. The presence of mutations within fragments of the genes encoding either a dihydropteroate synthase (DHPS) that plays a role in conferring resistance to sulfamethoxazole or a dihydrofolate reductase (DHFR) conferring resistance to trimethoprim in S. pneumoniae were tested by PCR followed by DNA sequence analysis [22]. Furthermore,
PCRs for the detection of two int-Tn genes encoding the integrases of the conjugative transposons $\operatorname{Tn} 1545$ (conferring resistance to tetracycline, kanamycin and macrolides) and Tn5252 (chloramphenicol resistance) were performed [21]. Amplicons of int-Tn1545 as well as intTn5252 were sequenced. Finally, capsular serotyping was conducted by the Quellung reaction [23] employing sera obtained from Statens Serum Institute (SSI), Copenhagen, Denmark. Typing sera included 12 serum pools for serogrouping and various type-specific antisera. Serotyping was performed according to the manufacturer's recommendations.

\section{Results}

All tested isolates were bile-soluble, optochin-susceptible and positive for the pneumolysin gene. MALDI-TOF MS identified all strains as S. pneumoniae. The recA and $16 \mathrm{~S}$ rDNA sequence analyses showed that all isolates were closely related to the type strain of S. pneumoniae. MLST revealed that all guinea pig isolates $(\mathrm{n}=6)$ belonged to sequence type (ST) 6937 (allelic profile 2-5-4-5-27-205), all equine isolates $(\mathrm{n}=3)$ were ST6934 (allelic profile 10-9-4-12-287-426-470), the dog and the two rat isolates were ST36 (allelic profile 1-8-4-1-1-4-6) and ST3546 (allelic profile 1-5-41-5-10-28-8), respectively. Susceptibility testing of the isolates showed that all but two isolates were susceptible to all antimicrobial agents tested. Isolates 2946 and 880, both obtained from rats, exhibited resistance to tetracycline, erythromycin, clindamycin, chloramphenicol and trimethoprim-sulfamethoxazole, and were positive for tet(M), erm $(\mathrm{B})$, cat $_{\mathrm{pC194}}$, and int-Tn1545 as well as int-Tn5252. Moreover, they displayed the same mutations in the genes sulA and $d f r$, i.e. an insertion of $6 \mathrm{bp}$ within sulA, the gene encoding DHPS, resulting in the duplication of amino acids Arg58 and Pro59 as well as mutations within the $d f r$ gene that resulted in amino acid exchanges at the position 92 (Asp92-Arg) and 100 (Ile-100-Leu) of the dihydrofolate reductase. These alterations have previously been described to be associated with sulfamethoxazole and trimethoprim resistance [24]. Serotyping identified all guinea pig isolates as serotype 19F, the three horse isolates as serotype 3 , the dog isolate as serotype $23 \mathrm{~F}$ and the rodent isolates as serotype $19 \mathrm{~A}$ (Table 1 ).

\section{Discussion}

A total of 12 non-repetitive S. pneumoniae isolates, originated from pet, companion animals and horses were analysed. The comparison of ST/serotype combination with the MLST database (http://pubmlst.org/spneumoniae/) showed the following outcomes. All six guinea pig isolates displayed a ST6937 and serotype 19F combination. Serotype $19 \mathrm{~F}$ is a common serotype encountered 
Table 1 Origin, molecular characterization, antimicrobial resistance and serotypes of the $12 \mathrm{~S}$. pneumoniae isolates investigated

\begin{tabular}{|c|c|c|c|c|c|c|c|c|}
\hline Isolate & Year of isolation & Host species & Site of isolation & Symptoms & ST & $\begin{array}{l}\text { Resistance phe- } \\
\text { notype }\end{array}$ & $\begin{array}{l}\text { Resistance geno- } \\
\text { type }\end{array}$ & Serotype \\
\hline 649 & 2009 & Guinea pig & Lung & Respiratory & 6937 & - & - & $19 F$ \\
\hline 2704 & 2009 & Horse & $\begin{array}{l}\text { Diverticulum tubae } \\
\text { auditivae }\end{array}$ & Respiratory & 6934 & - & - & 3 \\
\hline 2902 & 2009 & Horse & Trachea & Respiratory & 6934 & - & - & 3 \\
\hline 2946 & 2009 & Rat & Lung & Respiratory & 3546 & $\begin{array}{l}\text { TET, ERY, CLI, CHL, } \\
\text { SXT }\end{array}$ & $\begin{array}{l}\text { tet(M), erm(B), } \\
\text { cat }_{\mathrm{PC} 194}, \mathrm{dfr}\end{array}$ & $19 \mathrm{~A}$ \\
\hline 747 & 2010 & Horse & Trachea & Respiratory (RAO) & 6934 & - & - & 3 \\
\hline 1166 & 2010 & Guinea pig & Lung & $\begin{array}{l}\text { Central nervous, } \\
\text { respiratory }\end{array}$ & 6937 & - & - & $19 F$ \\
\hline 1409 & 2010 & Guinea pig & Lung & Respiratory & 6937 & - & - & $19 F$ \\
\hline 864 & 2011 & Guinea pig & Lung & Respiratory & 6937 & - & - & $19 F$ \\
\hline 2994 & 2012 & Guinea pig & Pleural punction & Respiratory & 6937 & - & - & $19 F$ \\
\hline 1537 & 2014 & Dog & Cerebrospinal fluid & Central nervous & 36 & - & - & $23 \mathrm{~F}$ \\
\hline 271 & 2017 & Guinea pig & Ear & Central nervous & 6937 & - & - & $19 F$ \\
\hline 880 & 2017 & Rat & Lung & Respiratory & 3546 & $\begin{array}{l}\text { TET, ERY, CLI, CHL, } \\
\text { SXT }\end{array}$ & $\begin{array}{l}\text { tet(M), erm(B), } \\
\text { cat }_{\mathrm{PC} 194,}, \mathrm{dfr}\end{array}$ & $19 \mathrm{~A}$ \\
\hline
\end{tabular}

in human isolates; according to the MLST database, which contains both ST and serotypes, it is associated with a large number of different STs and is often linked with invasive pneumococcal disease as well as S. pneumoniae carriage. This ST/serotype combination has actually 21 entries in the database, including 19 isolates from guinea pigs in Germany, the Netherlands, France and Peru. Another two isolates were found in a nasal and wound swab from infants in Germany and the Netherlands. It seems to be a typical ST/serotype combination among guinea pigs and this observation might suggest that guinea pigs seem to represent a reservoir for $S$. pneumoniae of this specific sequence type [25] with a still not clearly defined relevance in human hosts. Nevertheless, the close contact between children and their pets could be a risk factor for transmission. Our data also showed that this ST/serotype combination can be pathogenic for guinea pigs (Table 1 ).

The three equine isolates belong to ST6934 and serotype 3 . Serotype 3 is also a serotype commonly found in humans, but in the combination with ST6934, six entries were identified in the MLST database. All but one of the isolates originated from horses in Germany and the UK. Whether this particular serotype plays a role as a zoonotic pathogen remains unclear. Whatmore et al. [9] compared a collection of equine and human pneumococcal isolates of serotype 3 using restriction fragment length polymorphism (RFLP) analysis of housekeeping genes. RFLP revealed that equine pneumococci were indistinguishable from each other but different from human isolates. Thus, the results of the present study are in concordance with previous reports suggested that equine $S$. pneumoniae isolates likely represent a hostrestricted subpopulation of pneumococci.

Strain 1537/14 was isolated from a dog suffering from encephalitis, belonging to ST36 and serotype 23F. This $\mathrm{ST} /$ serotype combination has apparently not previously been reported from a dog or another animal. In the $S$. pneumoniae MLST database, S. pneumoniae ST36/23F is represented by 92 records, which all originate from human hosts. Serotype $23 \mathrm{~F}$ is one of the most prevalent serotypes involved in IPD [26].

The multidrug-resistant isolates 2946 and 880 originated from rats, kept as pet animals, that suffered from pneumonia. They belonged to ST3546 and serotype 19A. The MLST database revealed five records with this combination. Four of the isolates with this ST/serotype combination were isolated from humans in Germany, Norway and Czech Republic. One isolate was also obtained from a rat in Austria in 2007 with a similar multi-drug resistance profile. Linden et al. [25] also found two pet rats with the same ST/serotype combination. Rats are often kept by teenagers in close physical contact and suffer from pneumonia resistant to therapy [27]. It is recommended to examine those pets for the carriage of bacterial pathogens and-if positive-also by antimicrobial susceptibility testing more frequently than it is currently done. Serotype 19A is a common serotype found in humans suffering from IPD [28]. The epidemiology of this serotype is constantly changing. Possible reasons for this are the introduction of pneumococcal conjugate vaccination, increased use of antibiotics, import of 
multidrug-resistant isolates and increased reporting. The prevalence of serotype 19A, for example in Germany, has increased significantly between 2007 and 2011 [29].

Serogroup $19(12.8 \%)$ as well as serogroup $3(8.6 \%)$ are some of the most prevalent serogroups in humans in Europe. Among the most commonly reported serogroups, dual non-susceptibility to penicillin and macrolides was mainly observed in serogroup 19 . Serotypes $19 \mathrm{~F}$ and 23F, which were detected during the present study from guinea pigs and a dog, are known as common 'classic' resistant serotypes [30].

The isolates found in dogs and eventually in rats seem to be associated with human pneumococcal isolates, but further research is needed to improve the knowledge of the zoonotic potential of these bacteria. All companion animals considered in this study, suffered from severe respiratory or central nervous symptoms (Table 1). This strongly suggests that pneumococci are able to cause serious diseases in different animal species.

We recognize that the results of our study have limitations, because the examined collection comprises only twelve isolates. However, the frequency of S. pneumoniae isolation is in approximate concordance with previously described by van der Linden [25], who identified 41 strains during 22 years from pets and zoo animals.

\section{Conclusions}

Due to the close contact between companion animals and humans, isolation of human-associated clones in diseased companion animals deserves special consideration, particularly if those isolates display multidrug resistant pheno- and genotypes. The results of the present study confirm that a closer collaboration between human medicine and veterinary medicine is needed.

\section{Abbreviations}

CLSI: clinical and laboratory standards institute; DHFR: dihydrofolate reductase; DHPS: dihydropteroate synthase; IPD: invasive pneumococcal disease; MALDI-TOF: matrix-assisted laser desorption-ionization-time of flight; MLST: multilocus sequence typing; PCR: polymerase chain reaction; RFLP: restriction fragment length polymorphism; ST: sequence type.

\section{Authors' contributions}

MG performed the PCRs, microbiological testing and drafted the manuscript. ML, FK and DK participated in the planning of the study, data entry and sample collection. CM and GS participated in the planning of the study, performed and interpreted serological typing. IE participated in set up the molecular methods. ATF and SS participated in the planning and performing of the susceptibility testing, participated in drafting the manuscript. JS participated in the planning of the study and drafted the manuscript. IL had the original idea for the study, participated in microbiological and molecular biological testing and drafted the manuscript. All authors read and approved the final manuscript.

\section{Author details}

${ }^{1}$ Department of Pathobiology, Institute of Microbiology, University of Veterinary Medicine Vienna, Vienna, Austria. ${ }^{2}$ Department for Companion Animals and Horses, University of Veterinary Medicine Vienna, Vienna,
Austria. ${ }^{3}$ National Reference Laboratory for Pneumococcus, Austrian Agency for Health and Food Safety, Graz, Austria. ${ }^{4}$ The Healthcare Company of Styria, Graz, Austria. ${ }^{5}$ Centre of Infection Medicine, Department of Veterinary Medicine, Institute of Microbiology and Epizootics, Freie Universität Berlin, Berlin, Germany.

\section{Competing interests}

The authors declare that they have no competing interests.

\section{Availability of data and materials}

The strains and/or datasets generated and analysed during the current study are available on request to the corresponding author. Strain information has been deposited in the MLST Database (http://pubmlst.org/spneumoniae/).

\section{Consent for publication}

Not applicable.

\section{Ethics approval and consent to participate}

The examination of animal samples was carried out as part of routinely bacteriological diagnostic activity of the Institute of Microbiology. Therefore, according to Good Scientific Practice of the University of Veterinary Medicine, Vienna, this examination was not subject to the reporting obligation of the Ethics and Animal Welfare Commission of the University of Veterinary Medicine, Vienna.

\section{Funding}

This study was supported by internal funding of the participating institutes.

Prior publication of data

Data have not been published previously.

\section{Publisher's Note}

Springer Nature remains neutral with regard to jurisdictional claims in published maps and institutional affiliations.

Received: 27 August 2017 Accepted: 7 November 2017

Published online: 14 November 2017

\section{References}

1. Gillespie SH. Aspects of pneumococcal infection including bacterial virulence, host response and vaccination. J Med Microbiol. 1989;28:237-48. https://doi.org/10.1099/00222615-28-4-237.

2. Adriani KS, Brouwer MC, van der Ende A, van de Beek D. Bacterial meningitis in pregnancy: report of six cases and review of the literature. Clin Microbiol Infect. 2012;18:345-51. https://doi. org/10.1111/j.1469-0691.2011.03465.x.

3. Moxon ER. Experimental Infections of animals in the study of Streptococcus pneumoniae. Rev Infect Dis. 1981;3:354-7.

4. Chiavolini D, Pozzi G, Ricci S. Animal models of Streptococcus pneumoniae disease. Clin Microbiol Rev. 2008;21:666-85. https://doi.org/10.1128/ CMR.00012-0810.1093/clinids/3.2.354.

5. Duarte RS, Barros RR, Facklam RR, Teixeira LM. Phenotypic and genotypic characteristics of Streptococcus porcinus isolated from human sources. J Clin Microbiol. 2005;2005(43):4592-601. https://doi.org/10.1128/ JCM.43.9.4592-4601.2005.

6. Kuusi M, Lahti E, Virolainen A, Hatakka M, Vuento R, Rantala L, et al. An outbreak of Streptococcus equi subspecies zooepidemicus associated with consumption of fresh goat cheese. BMC Infect Dis. 2006;6:36. https://doi. org/10.1186/1471-2334-6-36.

7. Michaud S, Duperval R, Higgins R. Streptococcus suis meningitis: first case reported in Quebec. Can J Infect Dis. 1996;7:329-31.

8. Higgins R. Bacteria and fungi of marine mammals: a review. Can Vet J. 2000:41:105-16.

9. Whatmore AM, King SJ, Doherty NC, Sturgeon D, Chanter N, Dowson CG. Molecular characterization of equine isolates of Streptococcus pneumoniae: natural disruption of genes encoding the virulence factors pneumolysin and autolysin. Infect Immun. 1999;67:2776-82. 
10. Hakenbeck R, Balmelle N, Weber B, Gardès C, Keck W, de Saizieu A. Mosaic genes and mosaic chromosomes: intra- and interspecies genomic variation of Streptococcus pneumoniae. Infect Immun. 2001;69:2477-86. https://doi.org/10.1128/IAl.69.4.2477-2486.2001.

11. Mundy LS, Janoff EN, Schwebke KE, Shanholtzer CJ, Willard KE. Ambiguity in the identification of Streptococcus pneumoniae. Optochin, bile solubility, quellung, and the AccuProbe DNA probe tests. Am J Clin Pathol. 1998;109:55-61.

12. Zbinden A, Köhler N, Bloemberg GV. recA-based PCR Assay for accurate differentiation of Streptococcus pneumoniae from other viridans streptococci. J Clin Microbiol. 2011;49:523-7. https://doi.org/10.1128/ JCM.01450-10.

13. Loncaric I, Kübber-Heiss A, Posautz A, Stalder GL, Hoffmann D, Rosengarten $\mathrm{R}$, et al. Characterization of methicillin-resistant Staphylococcus spp. carrying the mecC gene, isolated from wildlife. J Antimicrob Chemother. 2013;68:2222-5. https://doi.org/10.1093/jac/dkt186.

14. Toikka P, Nikkari S, Ruuskanen O, Leinonen M, Mertsola J. Pneumolysin PCR-based diagnosis of invasive pneumococcal infection in children. Clin Microbiol. 1999;37:633-7.

15. Adamiak P, Vanderkooi OG, Kellner JD, Schryvers AB, Bettinger JA, Alcantara J. Effectiveness of the standard and an alternative set of Streptococcus pneumoniae multi locus sequence typing primers. BMC Microbiol. 2014;14:143. https://doi.org/10.1186/1471-2180-14-143.

16. Clinical and Laboratory Standards. Performance standards for antimicrobial disk and dilution susceptibility tests for bacteria isolated from animals. 3rd ed. Wayne: CLSI; 2015 (CLSI document VET01S-Ed3).

17. Clinical and Laboratory Standards Institute. Performance standards for antimicrobial susceptibility testing: twenty-seventh informational supplement. Wayne: CLSI; 2017.

18. Ambrose KD, Nisbet R, Stephens DS. Macrolide efflux in Streptococcus pneumoniae is mediated by a dual efflux pump (mel and mef) and is erythromycin inducible. Antimicrob Agents Chemother. 2005;49:4203-9. https://doi.org/10.1128/AAC.49.10.4203-4209.2005.

19. Maskell JP, Sefton AM, Hall LMC. Multiple mutations modulate the function of dihydrofolate reductase in trimethoprim-resistant Streptococcus pneumoniae. Antimicrob Agents Chemother. 2001;45:1104-8. https://doi. org/10.1128/AAC.45.4.1104-1108.2001.

20. Schnellmann C, Gerber V, Rossano A, Jaquier V, Panchaud Y, Doherr MG, et al. Presence of new mecA and $m p h(C)$ variants conferring antibiotic resistance in Staphylococcus spp. isolated from the skin of horses before and after clinic admission. J Clin Microbiol. 2006;44:4444-54. https://doi. org/10.1128/JCM.00868-06.

21. Shiojima T, Fujiki Y, Sagai H, lyobe S, Morikawa A. Prevalence of Streptococcus pneumoniae isolates bearing macrolide resistance genes in association with integrase genes of conjugative transposons in Japan. Clin Microbiol Infect. 2005;1 1:808-13. https://doi. org/10.1111/j.1469-0691.2005.01232.x.

22. Padayachee T, Klugman KP. Novel expansions of the gene encoding dihydropteroate dynthase in trimethoprim-sulfamethoxazole-resistant Streptococcus pneumoniae. Antimicrob Agents Chemother. 1999;43:2225-30.

23. Sørensen UB. Typing of pneumococci by using 12 pooled antisera. J Clin Microbiol. 1993;31:2097-100.

24. Cornick JE, Harris SR, Parry CM, Moore MJ, Jassi C, Kamng'ona A, et al. Genomic identification of a novel co-trimoxazole resistance genotype and its prevalence amongst Streptococcus pneumoniae in Malawi. J Antimicrob Chemother. 2014;69:368-74. https://doi.org/10.1093/jac/dkt384.

25. van der Linden M, Al-Lahham A, Nicklas W, Reinert RR. Molecular characterization of pneumococcal isolates from pets and laboratory animals. PLOS ONE. 2009;4(12):e8286. https://doi.org/10.1371/journal. pone.0008286.

26. Johnson HL, Deloria-Knoll M, Levine OS, Stoszek SK, Hance LF, Reithinger $R$, et al. Systematic evaluation of serotypes causing invasive pneumococcal disease among children under five: the pneumococcal global serotype project. PLOS Med. 2010;7(10):e1000348. https://doi.org/10.1371/ journal.pmed.1000348.

27. Weisbroth SH, Kohn DF, Boot R. Bacterial, mycoplasmal and mycotic infections-Chapter 11. In:Suckow MA, Weisbroth SH, Franklin CL, editors. The laboratory rat. 2nd ed. San Diego:Academic Press; 2005. p. 339-421.

28. Riva E, Salvini F, Garlaschi ML, Radaelli G, Giovannini M. The status of invasive pneumococcal disease among children younger than 5 years of age in north-west Lombardy, Italy. BMC Infect Dis. 2012;12:106. https:// doi.org/10.1186/1471-2334-12-106.

29. Linden $M$, van der Reinert RR, Kern WV, Imöhl M. Epidemiology of serotype 19A isolates from invasive pneumococcal disease in German children. BMC Infect Dis. 2013;13:70. https://doi. org/10.1186/1471-2334-13-70.

30. European Centre for Disease Prevention and Control. Antimicrobial resistance surveillance in Europe 2014. Annual Report of the European Antimicrobial Resistance Surveillance Network (EARS-Net). Stockholm: ECDC; 2015.

\section{Submit your next manuscript to BioMed Central and we will help you at every step:}

- We accept pre-submission inquiries

- Our selector tool helps you to find the most relevant journal

- We provide round the clock customer support

- Convenient online submission

- Thorough peer review

- Inclusion in PubMed and all major indexing services

- Maximum visibility for your research

Submit your manuscript at www.biomedcentral.com/submit
() BioMed Central 